\title{
Transcriptional regulation of the biosynthesis of phytoalexin: A lesson from specialized metabolites in rice
}

\author{
Koji Miyamoto', Takafumi Shimizu², Kazunori Okada ${ }^{3, *}$ \\ ${ }^{1}$ Department of Biosciences, Teikyo University, Utsunomiya, Tochigi 320-8551, Japan; ${ }^{2}$ Center for Sustainable Resource \\ Science, RIKEN, Yokohama, Kanagawa 230-0045, Japan; ${ }^{3}$ Biotechnology Research Center, The University of Tokyo, Bunkyo- \\ ku, Tokyo 113-8657, Japan \\ *E-mail: ukokada@mail.ecc.u-tokyo.ac.jp Tel: +81-35-841-3070
}

Received July 4, 2014; accepted July 30, 2014 (Edited by T. Shoji)

\begin{abstract}
Plants have the ability to produce vast amounts of secondary metabolites, including terpenoids, flavonoids, alkaloids, and their derivatives. These metabolites sometimes affect the growth of other organisms, either positively or negatively. Production of defensive compounds, for example phytoalexins, is a well-known strategy used by plants to defend against and withstand invading pathogens. As phytoalexins serve an obvious function in plant defense, they are known as specialized metabolites, substances with a specific biological role that function during specific circumstances. In rice, 15 diterpenoid-type phytoalexins and one flavonoid-type phytoalexin have been identified. Extensive studies have explored the biochemistry, biosynthesis, and biological functions of phytoalexins in rice, specifically the mechanism by which they function in disease resistance. This review focuses on our current knowledge of the transcriptional and hormonal regulation of the production of phytoalexins in rice.
\end{abstract}

Key words: Flavonoid, jasmonate, rice, terpenoid, transcription factor.

\section{Phytoalexins as defensive compounds}

The disease resistance of rice, a staple crop, has been extensively investigated. The production of secondary metabolites called phytoalexins, which function in defense, was discovered in rice leaves that had been exposed to the rice blast Magnaporthe oryzae over 50 years ago. An important event triggering phytoalexin production is the perception of a chitin oligosaccharide, one of the microbe-associated molecular patterns (MAMPs) from the blast fungus, by specific receptors (CEBiP and CERK) (Shimizu et al. 2010). The production of phytoalexins has been induced not only by treating rice cells with chitin elicitor but also by exposing rice to ultraviolet (UV) irradiation, phytohormones, cholic acid, and heavy metals such as $\mathrm{CuCl}_{2}$ (Daw et al. 2008; Ko et al. 2010; Koga et al. 2006; Nakazato et al. 2000; Rakwal et al. 1996b; Shimizu et al. 2008).

The rice phytoalexins that have been identified fall into two major groups: the first group includes 15 diterpenoid-type phytoalexins: momilactones $\mathrm{A}$ and
B (Cartwright et al. 1981), oryzalexin A-F (Akatsuka et al. 1983; Akatsuka et al. 1985; Kato et al. 1993; Kato et al. 1994; Kono et al. 1984; Kono et al. 1985; Sekido et al. 1986), oryzalexin S (Kodama et al. 1992b), phytocassanes A-E (Koga et al. 1995; Koga et al. 1997; Yajima and Mori 2000), which are collectively known as labdane-related diterpenoids, and ent-10-oxodepressin, a macrocyclic diterpenoid (Inoue et al. 2013). The second group includes a single phytoalexin, the flavonoidtype phytoalexin sakuranetin (Kodama et al. 1992a). Among these, momilactones, phytocassanes, ent-10oxodepressin and sakuranetin are considered biologically important phytoalexins because they show high antimicrobial activity in in vitro assays, demonstrating potent inhibitory activity on germ tube elongation of $M$. oryzae, with an $\mathrm{ED}_{50}$ value below $1-10 \mathrm{mg} \cdot \mathrm{l}^{-1}$ (Cartwright et al. 1977; Inoue et al. 2013). They also show high accumulation in rice leaves infected by $M$. oryzae. It has recently been shown that momilactone A actually inhibits the growth of rice blast fungus at the site of infection in rice leaves (Hasegawa et al. 2010). In

Abbreviations: BTH, benzothiadiazole; bZIP, basic leucine zipper; CK, cytokinin; COMT, caffeic acid 3-O-methyltransferase; CYP, cytochrome P450; DMAPP, dimethulallyl diphosphate; ET, ethylene; GGDP, geranylgeranyl diphosphate; IPP, isopentenyl diphosphate; JA, jasmonic acid; JA-Ile, jasmonoyl-isoleucine; MAMPs, microbe-associated molecular patterns; MEP, methylerythritol phosphate; MVA, mevalonate; NOMT, naringenin 7-O-methyltransferase; PAL, phenylalanine ammonia lyase; RNAi, RNA interference; SA, salicylic acid; UV, ultraviolet.

This article can be found at http://www.jspcmb.jp/

Published online November 22, 2014 
Diterpenoid type phytoalexins

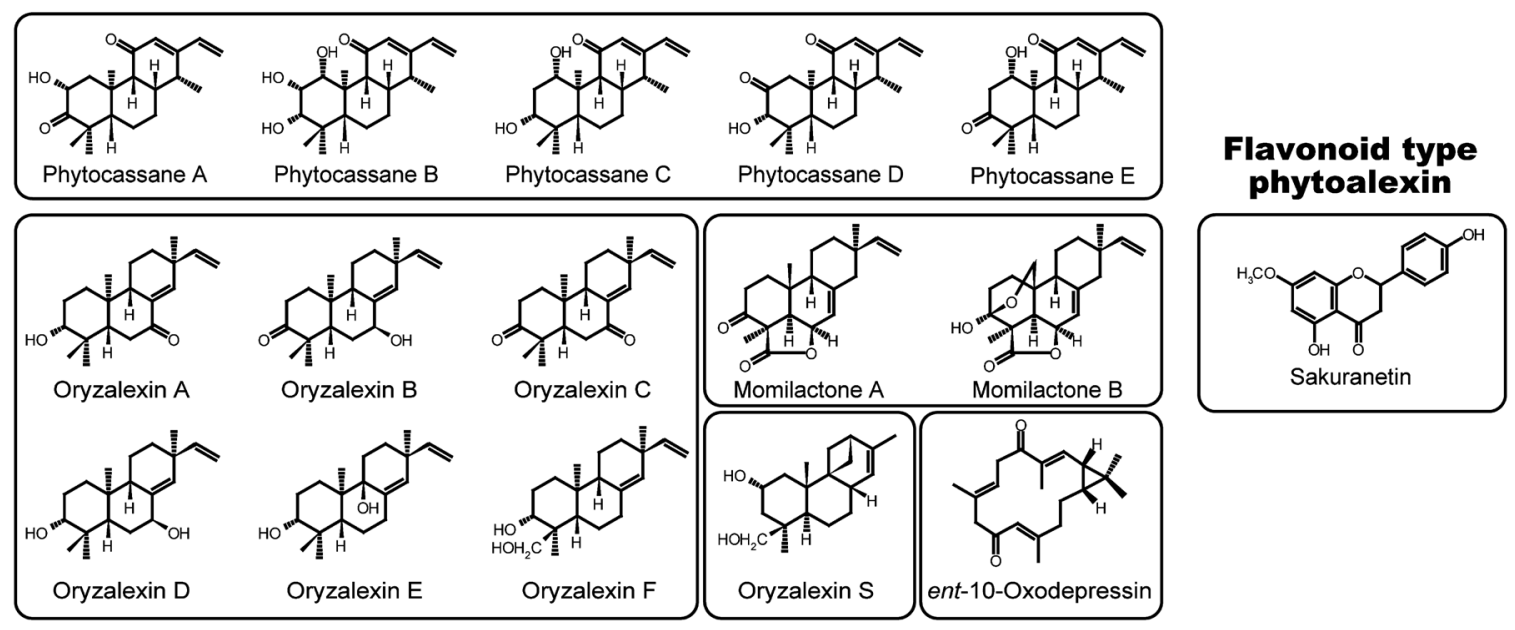

Figure 1. Known phytoalexins in rice. Of the 16 phytoalexins identified in rice, all are diterpenoids except for the flavonoid sakuranetin. Diterpenoid phytoalexins are classified into five groups according to the structure of their hydrocarbon precursors: phytocassanes A through E, momilactones A and B, oryzalexin A through F, oryzalexin S, and ent-10-oxodepressin.

contrast, there is no direct evidence that phytocassanes, sakuranetin, and other types of phytoalexins prevent fungal growth at the site of infection in rice.

Rice also constitutively produces phytoalexins in roots and exudes them into the rhizosphere (Toyomasu et al. 2008). Momilactone B is an allelopathic substance exuded from the roots of rice that inhibits the growth of neighboring plants (Kato-Noguchi and Ino 2003). Phytocassanes are also constitutively exuded with momilactones; they are believed to be defensive substances, but not phytotoxic substances (Toyomasu et al. 2008). Given that the production of phytoalexins is constitutive or induced and that they play defensive roles, it appears that rice has evolved the production of phytoalexins as specialized metabolites. Their characteristic biosynthetic routes rely on the expression of relevant biosynthetic genes, some of which are located close together on chromosomes in genomic clusters.

\section{Biosynthetic pathways and genes for diterpenoid phytoalexins}

In plants, isopentenyl diphosphate (IPP) and dimethylallyl diphosphate (DMAPP), which are the basic C5 precursors for terpenoid biosynthesis, are produced via two distinct pathways: the mevalonate (MVA) pathway and the methylerythritol phosphate (MEP) pathway (Lichtenthaler et al. 1997). Sesquiterpenes and triterpenes are synthesized in the cytoplasm from IPP that is derived from the MVA pathway (Vranová et al. 2013). In contrast, diterpenes, including diterpenoid phytoalexins, are synthesized in plastids from IPP and DMAPP that are derived from the MEP pathway (Estévez et al. 2001; Vranová et al. 2013) (Figure 2). In fact, the elicitor-induced accumulation of diterpenoid phytoalexin in rice cells is suppressed by treatment with 5-ketoclomazone and fosmidomycin, chemical inhibitors of MEP pathway, but not by treatment with mevastatin, an inhibitor of the MVA pathway (Okada et al. 2007). In addition, the expression of seven MEP pathway genes (OsDXS3, OsDXR, OsCMS, OsCMK, OsMCS, OsHDS, and $O s H D R)$ is synchronously induced in elicited rice cells and rice leaves treated with jasmonic acid (JA), UV, or $\mathrm{CuCl}_{2}$. This effect provides further evidence that the MEP pathway is involved in inducible production of diterpene phytoalexins (Okada et al. 2007).

In the phytoalexin biosynthesis, geranylgeranyl diphosphate (GGDP), a common precursor of diterpenoids, is converted into diterpene hydrocarbons in two sequential cyclization steps. Six diterpene cyclase involved in these steps have been identified (Peters 2006; Toyomasu 2008). GGDP is first cyclized by OsCPS2/ OsCyc2 and OsCSP4/OsCyc1 to form ent-copalyl diphosphate and syn-copalyl diphosphate (Otomo et al. 2004b; Prisic et al. 2004; Sakamoto et al. 2004; Xu et al. 2004). Next, OsKSL7/OsDTC1, OsKSL10, OsKSL4, and OsKSL8/OsDTC2 catalyze the second cyclization of entcopalyl diphosphate or syn-copalyl diphosphate to four distinct diterpene hydrocarbons: ent-cassa-12,15-diene, ent-sandaracopimaradiene, 9 $\beta \mathrm{H}$-pimara-7,15-diene, and stemar-13-ene (Cho et al. 2004; Nemoto et al. 2004; Otomo et al. 2004a; Wilderman et al. 2004) (Figure 2). In contrast, cDNA encoding a diterpene cyclase responsible for biosynthesis of ent-10-oxodepressin, a macrocyclic diterpenoid, has not been identified yet.

After the diterpene cyclases involved in diterpenoid phytoalexin biosynthesis were identified, it was shown that OsCPS4 and OsKSL4, which encode diterpene cyclases involved in momilactone biosynthesis, are located in a narrow region on rice chromosome 4 . 


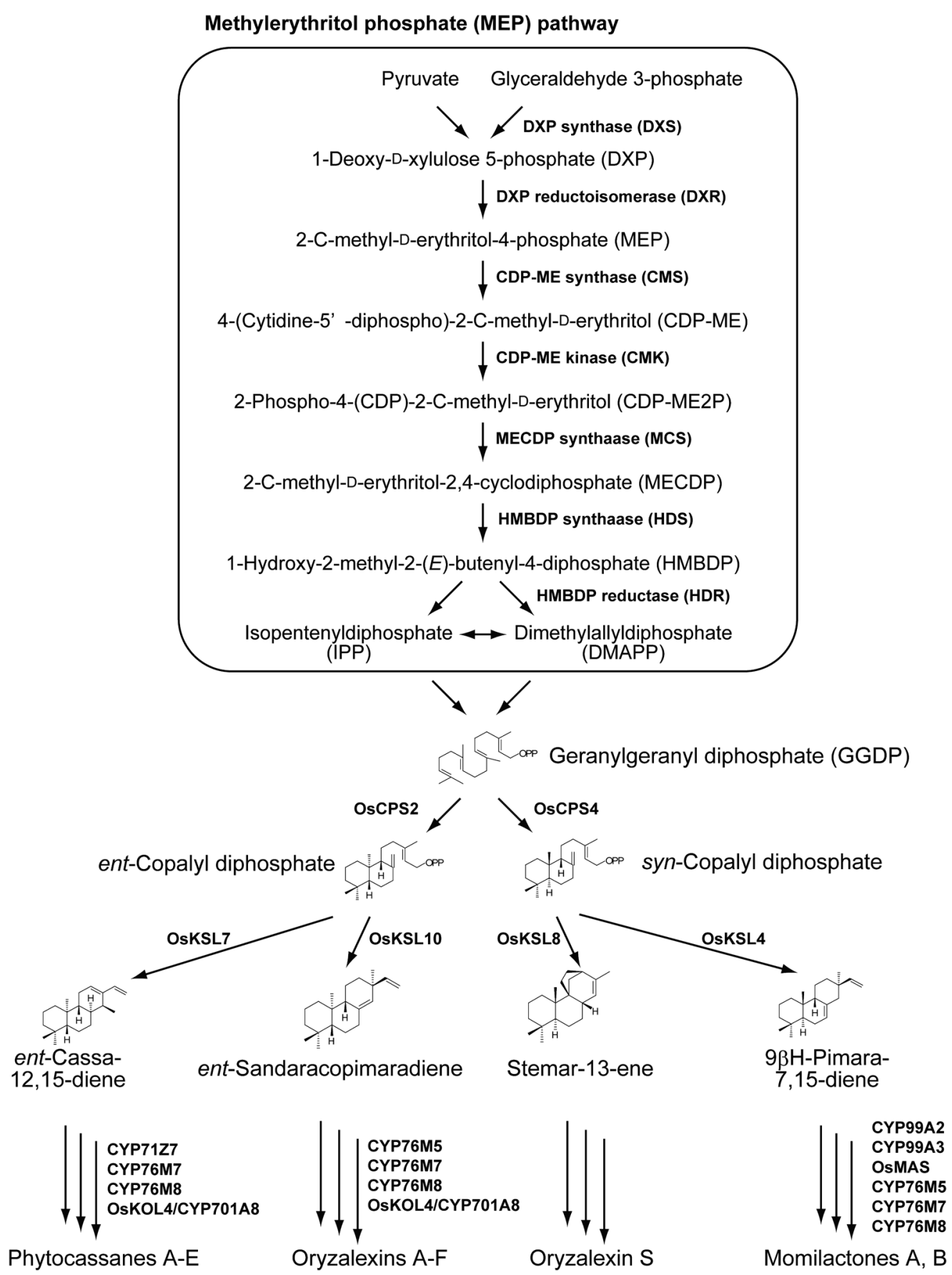

Figure 2. Established biosynthetic routes of diterpenoid phytoalexins. Diterpenoid phytoalexins are synthesized from geranylgeranyl diphosphate (GGDP). GGDP is cyclized by terpene cyclase (OsCPSs and OsKSLs) and then converted to each phytoalexin by P450 monooxygenase and dehydrogenase. Activation of the methylerythritol phosphate (MEP) pathway leading to GGDP synthesis may be required to supply sufficient amounts of terpenoid precursors for the production of phytoalexins.

Two cytochrome P450 (CYP) genes (CYP99A2 and CYP99A3) and a dehydrogenase gene are located in the same region, forming a gene cluster (Sakamoto et al. 2004). Early work with cell-free extracts suggested that CYPs and dehydrogenases are involved in the downstream oxidation steps of diterpene hydrocarbon precursors (Atawong et al. 2002; Kato et al. 1995). The following line of evidence clearly proved that assumption. Double knockdown of CYP99A2 and CYP99A3 by
RNA interference (RNAi) caused the reduction of momilactone accumulation in elicitor-treated rice cells (Shimura et al. 2007). Moreover, CYP99A3 catalyzed C19-oxidation of $9 \beta \mathrm{H}$-pimara-7,15-diene in vitro, and $9 \beta \mathrm{H}$-pimara-7,15-diene-19-oic acid was detected in rice plants, suggesting that CYP99A2 and/or CYP99A3 are involved in the downstream oxidation steps of the diterpene hydrocarbons (Wang et al. 2011). The function of the dehydrogenase gene as a momilactone A synthase 
(OsMAS) was also investigated by in vitro enzyme assay, resulting in the catalytic conversion of $3 \beta$-hydroxy$9 \beta \mathrm{H}$-pimara-7,15-diene-19,6 $\beta$-olide into momilactone A (Shimura et al. 2007). Taken together, these reports indicate the presence of a momilactone biosynthetic gene cluster, although further research is required to reveal the complete pathway for momilactone biosynthesis.

The phytocassane biosynthetic genes OsCPS2 and OsKSL7 are located in a narrow region on rice chromosome 2, along with two diterpene cyclase genes (OsKSL5 and OsKSL6) and six CYP genes (CYP71Z6, CYP71Z7, and CYP76M5-8), creating another gene cluster. These five CYP genes, with the exception of CYP71Z6, show an elicitor-inducible expression pattern similar to that of OsCPS2 and OsKSL7 (Okada et al. 2007), suggesting that these CYP genes are involved in the biosynthesis of phytocassane.

In recent years, Reuben Peters' group has extensively studied and summarized the catalytic functions of these CYPs using in vitro enzyme assay, as described in their recent review (Schmelz et al. 2014). It was reported that CYP76M7 catalyzes the $11 \alpha$-hydroxylation of ent-cassa12,15-diene (Swaminathan et al. 2009). In addition, CYP71Z7 can catalyze the C2-hydroxylation of ent-cassa12,15 -diene in vitro. However, ent-cassa-12,15-diene is a poorly recognized substrate for CYP71Z7 $\left(K_{\mathrm{m}}=200 \mu \mathrm{M}\right)$ in in vitro assays, suggesting that the true substrate of CYP71Z7 in planta has not been identified (Wu et al. 2011).

We used a transgenic approach to investigate the involvement of these CYPs in the biosynthesis of phytocassane. Double knockdown of CYP76M7 and CYP76M8 by RNAi causes a decrease in phytocassane accumulation in elicitor-treated rice cells. Collectively, these biochemical and genetic data indicate that CYP76M7 and/or CYP76M8 are involved in phytocassane biosynthesis (Wang et al. 2012a). We also found that the accumulation of $\mathrm{C} 2$-oxygenated phytocassanes is specifically suppressed in CYP71Z7 knockdown lines (Okada 2011). Taken together, these reports indicate the presence of the phytocassane biosynthetic gene cluster. However, it has also been reported that another CYP, which is encoded out of the gene cluster, is involved in phytocassane biosynthesis. OsKOL4/CYP701A8, an ent-kaurene oxidase paralog encoded on rice chromosome 6 , catalyzes the $3 \alpha$-hydroxylation of ent-cassa-12,15-diene and entsandaracopimaradiene, suggesting that this enzyme functions in the biosynthesis of both phytocassane and oryzalexin (Wang et al. 2012b).

In vitro conversion assays of diterpene hydrocarbons other than ent-cassa-12,15-diene with CYP76M5, CYP76M6, and CYP76M8 exhibited promiscuous catalytic activities, resulting in the $6 \beta$-hydroxylation of syn-pimara-7,15-diene and $7 \beta$-hydroxylation of ent-sandaracopimaradiene (Wang et al. 2012a). The findings suggest that they serve potential functions in momilactone and oryzalexin biosynthesis (Figure 2).

Two genes (OsKSL6 and CYP71Z6) on the chromosome 2 gene cluster may be relevant to the biosynthesis of oryzalide-related diterpenoids, which are phytoanticipins produced by rice (Kono et al. 1991; Kono et al. 2004; Watanabe et al. 1990; Watanabe et al. 1992). OsKSL6 catalyzes the cyclization of ent-copalyl diphosphate to ent-kaur-15-ene, which is putative precursor of oryzalide-related compounds (Kanno et al. 2006). CYP71Z6 also catalyzes the C2-hydroxylation of ent-kaur-15-ene (Wu et al. 2011). These facts suggest that the chromosome 2 gene cluster may be associated not only with phytocassane biosynthesis but also with the biosynthesis of oryzalide-related compounds. It remains to be investigated whether the biosynthetic enzymes from the chromosome 2 gene cluster are involved in the biosynthesis of oryzalide-related compounds, momilactones, and oryzalexins in vivo.

\section{Gene clusters for the biosynthesis of specialized metabolites}

The momilactone and phytocassane biosynthetic genes are clustered as described, whereas the oryzalexin biosynthetic genes are not clustered. To date, over 10 examples of plant metabolic gene clusters for the biosynthesis of defense compounds have been reported (Nützmann and Osbourn 2014). It is known that plant metabolic gene clusters exhibit tissue- or site-specific co-expression patterns (Nützmann and Osbourn 2014). Therefore, it has been hypothesized that the formation of a gene cluster allows the coordinated regulation of clustered genes and the efficient biosynthesis of specialized metabolites (Chu et al. 2011). Indeed, the momilactone and phytocassane biosynthetic genes exhibit a root-specific expression pattern (Toyomasu et al. 2008). Moreover, these genes show the temporally coordinated expression of mRNAs after treatment with an elicitor in rice cells, resulting in synchronized production of momilactones and phytocassanes (Okada et al. 2007; Shimura et al. 2007).

It is possible that the clustering of the momilactone and phytocassane biosynthetic genes contributes to the effective production of these phytoalexins. Consistent with this idea, momilactones and phytocassanes accumulate at higher levels than does oryzalexin $\mathrm{S}$ in rice leaves infected with M. oryzae (Toyomasu et al. 2014). It has also been hypothesized that the formation of gene clusters facilitates the co-inheritance of functionally related biosynthetic genes. Deficits in biosynthetic genes for specialized metabolites can lead not only to a loss of the end product but also to the accumulation of toxic bioactive intermediates. The co-inheritance of 
functionally related biosynthetic genes may prevent the loss of parts of these genes. Further research to investigate the complete biosynthetic pathways of the diterpenoid phytoalexin and the transcriptional regulatory mechanism(s) of the momilactone and phytocassane biosynthetic gene clusters may reveal the significance of clustering of biosynthetic genes.

\section{Biosynthetic genes involved in sakuranetin production}

The flavonoid sakuranetin was first identified from Prunus bark cortex as an aglycone of sakuranin (Asahina 1908). After that, sakuranetin was found in rice and several other plant species, including Artemisia campestris, Prunus spp., Baccharis spp., Betula spp., and Juglans spp. (Ahuja et al. 2012). The final step in sakuranetin biosynthesis is 7-O-methylation of naringenin, a key biosynthetic intermediate in isoflavone and a variety of flavones, which is catalyzed by $S$-adenosyl-L-methionine dependent naringenin 7-O-methyltransferase (NOMT) (Rakwal et al. 1996a). Therefore, NOMT plays a key role in sakuranetin biosynthesis at the point at which it branches from a common flavonoid biosynthetic pathway.

The first purification of NOMT to apparent homogeneity from crude extracts of UV-treated wildtype rice leaves was likely accomplished by Rakwal et al. (2000). The purified protein, however, was actually highly homologous to a caffeic acid 3-O-methyltransferase (COMT) from maize, and in vitro enzyme assay consistently showed that it had COMT activity, but not NOMT activity. Thus, this enzyme was named OsCOMT1 (Lin et al. 1996; Rakwal et al. 2000).

As a loss-of-function mutant of OsCOMT1 was able to confer NOMT enzymatic activity and accumulation of sakuranetin after elicitation, OsCOMT1 apparently is not essential for sakuranetin production in rice. Therefore, we took advantage of the OsCOMT1 mutant to purify the OsNOMT enzyme and successfully obtained active OsNOMT protein, thereby identifying the OsNOMT gene in rice (Shimizu et al. 2012). An in vitro enzyme assay using a recombinant OsNOMT protein tagged with glutathione-S-transferase exhibited the highest substrate preference to naringenin with a reasonable $K_{\mathrm{m}}$ value $(1.9 \mu \mathrm{M})$, compared with other related substrates, the flavanone liquiritigenin and flavones. The protein showed no methylation activity on phenolic compounds, including isoflavonoids. As OsNOMT mRNA was induced prior to sakuranetin accumulation in elicited rice leaves, these results strongly suggested that OsNOMT is a major sakuranetin synthase (Shimizu et al. 2012).

Naringenin is commonly biosynthesized from phenylalanine via the phenylpropanoid and flavonoid pathways. According to the Rice Annotation Project database (RAP-DB: http://rapdb.dna.affrc.go.jp/), there are a number of homologous genes involved in the phenylpropanoid and flavonoid pathways, including 10 phenylalanine ammonia lyases (PAL), 11 4-coumarate CoA ligases, 13 chalcone synthases, and seven chalcone isomerases in the rice genome. Among these, OsPAL06 knock-down plant shows a lower accumulation of sakuranetin and naringenin in the roots, suggesting that OsPAL06 is involved in sakuranetin production in rice roots (Duan et al. 2014). However, the contribution of other genes in the phenylpropanoid and flavonoid pathway to stress-inducible sakuranetin production has not been demonstrated. Some of these genes show a co-expression pattern with OsNOMT in response to JA treatment or UV-irradiation (Miyamoto et al. 2012; Park et al. 2013). Such information might help to identify biosynthetic genes involved in the production of sakuranetin.

\section{Signaling pathways leading to the phytoalexin biosynthesis mediated by plant hormones}

An important topic of study is endogenous signaling leading to the induction of phytoalexin production after the perception of biotic and abiotic elicitors. It has been assumed that similar signaling pathways may induce both diterpenoid and flavonoid phytoalexin production in rice. However, current studies demonstrate that the signaling pathways for diterpenoid and flavonoid phytoalexin production have important differences.

One of the best studied signaling pathways involved in stress-induced phytoalexin production in rice is mediated by jasmonate, a plant hormone that is inductively synthesized in response to wounding and pathogen infections. A component of jasmonate, jasmonoyl-isoleucine (JA-Ile), is biosynthesized by conjugating isoleucine to JA by JAR1 protein. It is a major active compound perceived by the co-receptor complex of COI1, an F-box component of the SCFtype E3-ubiquitin ligase complex (SCF ${ }^{\mathrm{COI}}$ ), which triggers ubiquitination and subsequent degradation of JAZ repressor proteins by $26 \mathrm{~s}$ proteasome. Release of a transcriptional activator such as MYC2 from repression by the JAZ protein interaction accelerates the expression of downstream genes, such as JA-inducible defenserelated genes (Wasternack and Hause 2013). These core components in JA signaling are conserved in rice as well (Cai et al. 2014; Taniguchi et al. 2014a; Ye et al. 2012).

The production of rice diterpenoid phytoalexins and the flavonoid sakuranetin is induced not only by biotic and abiotic stresses, as mentioned above, but also by JA treatment in rice leaves (Rakwal et al. 1996a; Rakwal et al. 1996b). Jasmonate also induces expression of 
phytoalexin biosynthetic genes in rice leaves (Miyamoto et al. 2012). Furthermore, jasmonate accumulates in response to $M$. oryzae infection and $\mathrm{CuCl}_{2}$ treatment in rice leaves (Rakwal et al. 1996b; Riemann et al. 2013). In suspension-cultured rice cells, chitin elicitor treatment induces accumulation of JA, followed by accumulation of momilactone A (Nojiri et al. 1996). Hence, jasmonate is thought to be an important secondary signaling molecule for the production of the diterpenoid phytoalexins and sakuranetin in rice.

Recently, several rice jasmonate mutants were identified. Using them, researchers have investigated the contribution of endogenous jasmonate to phytoalexin production in detail. In two loss-of-function mutants of allene oxide cyclase of rice, cpm2 and hebiba, constitutive and wound-induced levels of JA and JA-Ile were extremely low. When leaves were inoculated with $M$. oryzae, the accumulation levels of momilactone B and phytocassanes were comparable in cpm2, hebiba, and the wild type, although accumulation of momilactone A was lower in cpm2 and hebiba than in the wild type. On the other hand, the accumulation of sakuranetin in leaves of cpm2 and hebiba that were inoculated with $M$. oryzae was severely suppressed compared with that of the wild type (Riemann et al. 2013). These results are inconsistent with the original understanding that jasmonate is an important secondary signaling molecule for the production of both diterpenoid phytoalexins and sakuranetin in rice. Of course, jasmonate has some impacts on the production of diterpenoid phytoalexins under specific conditions, but it is clearly not essential, whereas in sakuranetin production it is an absolute requirement.

Further investigation of phytoalexin production focusing on JA-Ile, an active jasmonate, was performed by using the rice JAR1 mutant osjar1-2, which contains remarkably lower levels of JA-Ile than the wild type after wounding. Several experiments have demonstrated the significance of JA-Ile. When leaves of osjar1-2 were treated with JA, accumulation levels of both sakuranetin and diterpenoid phytoalexins were severely suppressed compared with those of the wild type, but the response of osjar1-2 was restored by treatment with JA-Ile. On the other hand, when osjar1-2 was inoculated with $M$. oryzae or treated with $\mathrm{CuCl}_{2}$, the accumulation levels of sakuranetin were far lower than those of the wild type, whereas the levels of diterpenoid phytoalexins were comparable to those of the wild type, as has been observed in the cpm2 and hebiba mutants (Shimizu et al. 2013). These results suggested the existence of a JA-Iledependent pathway for production of both diterpenoid phytoalexins and sakuranetin, but a jasmonateindependent signaling pathway is also involved in stressinduced production of diterpenoid phytoalexins. Further analysis is necessary to find an endogenous signaling molecule capable of the production of diterpenoid phytoalexins in the rice jasmonate mutants, which in turn can provide evidence for a jasmonate-independent pathway for phytoalexin production.

Regarding core signaling components of JA-Ile, the rice genome encodes three copies of COI1 and 14 copies of JAZ protein genes. Among these, OsCOI1a and OsJAZ8 were individually reported to be involved in jasmonate-mediated rice defense responses (Taniguchi et al. 2014a; Taniguchi et al. 2014b; Ye et al. 2012). However, their roles in the production of rice phytoalexins have not been investigated. On the other hand, OsPAD4, a lipase-like protein, was suggested to be involved in JA accumulation, JA-mediated systemic defense responses, and the accumulation of momilactone $\mathrm{A}$ in rice. By comparison, Arabidopsis AtPAD4 functions in SAdependent defense signaling by interacting with another lipase-like protein and is involved in the production of camalexin (Ke et al. 2014). How OsPAD4 functions in rice is still unknown, but these results suggested that OsPAD4 may have a specific function for JA-mediated signaling in rice and may be involved in rice phytoalexin production (Figure 3A).

Treatment with natural and synthetic cytokinins (CKs), as well as with jasmonate, induces the production of diterpenoid phytoalexins in rice leaves and suspension-cultured cells (Ko et al. 2010). However, CK treatment inhibits JA-inducible sakuranetin production in rice leaves (Tamogami et al. 1997). On the other hand, exogenous root applications of salicylic acid (SA) promote the accumulation of oryzalexins and momilactone A in leaves (Daw et al. 2008). Recent reports presented the role of CKs and SA in plant defense responses (Choi et al. 2010; Großkinsky et al. 2011; Jiang et al. 2013). A synergistic crosstalk of CK/SA signaling was also reported showing that $0.1 \mathrm{mM}$ CKs treated together with benzothiadiazole (BTH), a plant activator that enhances SA signaling pathway, had severalfold enhancement of the induction of momilactone and phytocassane biosynthetic genes (Akagi et al. 2014). Besides, SA-dependent and JA-dependent pathways frequently interact either synergistically or antagonistically in plant-pathogen interaction (De Vleesschauwer et al. 2013) (Figure 3B, C).

In addition, it was reported that treatment of wounded rice leaves with methionine, the precursor of ethylene (ET), could induce the accumulation of momilactone A and sakuranetin. However, ET treatment of wounded leaves can induce sakuranetin but not momilactone A, suggesting that the induction of diterpenoid phytoalexin production by methionine is not regulated by ET alone (Nakazato et al. 2000; Xu et al. 2012) (Figure 3D). As mentioned, there are different views of the effects of plant hormone treatment on phytoalexin production in rice. The endogenous roles of each hormone remain 
A

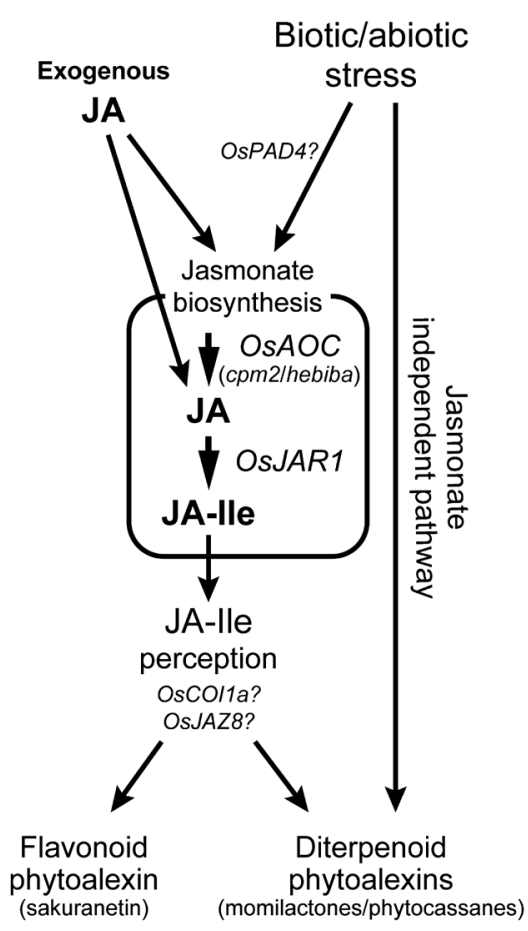

B

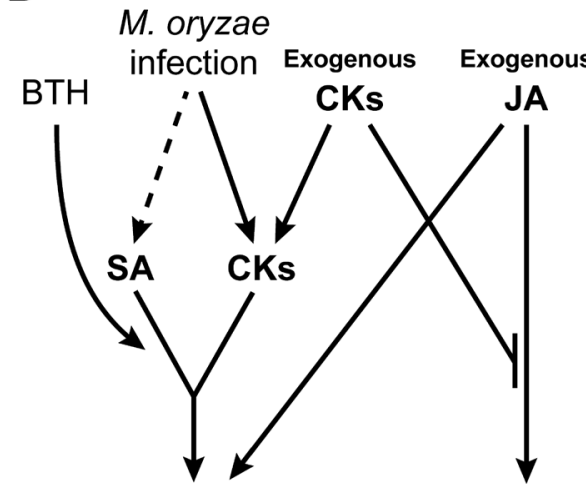

Diterpenoid phytoalexins loryzalexins)

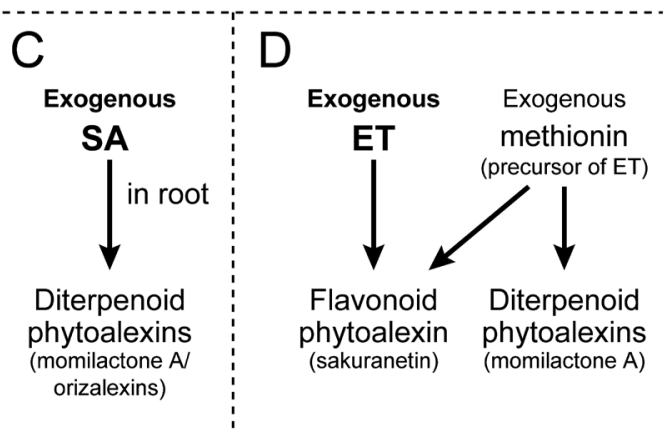

Figure 3. Hormonal regulation of phytoalexin production. (A) Role of jasmonate in phytoalexin production of rice. Endogenous jasmonoylisoleucine (JA-Ile) induced by biotic and abiotic stress is involved in the production of both flavonoid and diterpenoid phytoalexins. JA-Ile-mediated signaling is indispensable to the production of flavonoid phytoalexin. Production of diterpenoid phytoalexins also may be induced by a jasmonateindependent pathway. (B) Crosstalk among cytokinins (CKs), salicylic acid (SA), and jasmonate on phytoalexin production. Treatment with CKs induces production of diterpenoid phytoalexins in suspension-cultured rice cells and rice leaves. In response to Magnaporthe oryzae infection, CKs and SA synergistically induce the production of diterpenoid phytoalexins. Benzothiadiazole (BTH) acts as a plant activator that enhances SA signaling. On the other hand, treatment of CKs inhibits the production of JA-induced production of flavonoid phytoalexin in rice leaves. (C) Effect of exogenously applied salicylic acid (SA) on phytoalexin production in rice roots. SA treatment induces the production of diterpenoid phytoalexins in rice roots. (D) Effect of exogenously applied ethylene (ET) and methionine on phytoalexin production. Treatment with methionine, the precursor of ET, induces production of both flavonoid and diterpenoid phytoalexins in rice leaves, whereas treatment with ET alone induces only the production of flavonoid phytoalexin in rice leaves.

to be elucidated. In the future, investigations of the roles not only of endogenous jasmonate but also of the other endogenous hormones, such as SA, ET, and CKs, are necessary in order to fully understand regulation of stress-induced phytoalexin production in rice.

\section{Transcriptional networks regulating the gene expression for phytoalexin production}

Much work has been done in an effort to identify the transcription factors involved in the regulation of diterpenoid phytoalexin production. Transcription factors that are inductively expressed during the blast infection or simply treated with chitin elicitor have been screened in an attempt to find candidate factors by using transcriptomes. Some of these were not responsive to blast infection, probably due to the infectious agent's organ-specific effects, whereas the chitin elicitor treatment of rice cells can trigger the expression of all factors identified. The transcriptional network comprised of such transcription factors involved in the phytoalexin biosynthesis is summarized in Figure 4.

Okada et al. (2009) reported that the chitin elicitorinduced basic leucine zipper (bZIP) transcription factor OsTGAP1 is involved in the regulation of the production of momilactones and phytocassanes in elicitor-treated rice cells. OsTGAP1-overexpressing (OsTGAP1ox) rice cells exhibit hyperaccumulation of momilactones and phytocassanes, as well as enhanced expression of all momilactone biosynthetic genes, the phytocassane biosynthetic gene OsKSL7, and the MEP pathway gene OsDXS3, after being treated with an elicitor (Okada et al. 2009). We sought to investigate the mechanisms of the elicitor-stimulated coordinated hyperinduction of these phytoalexin biosynthetic genes 


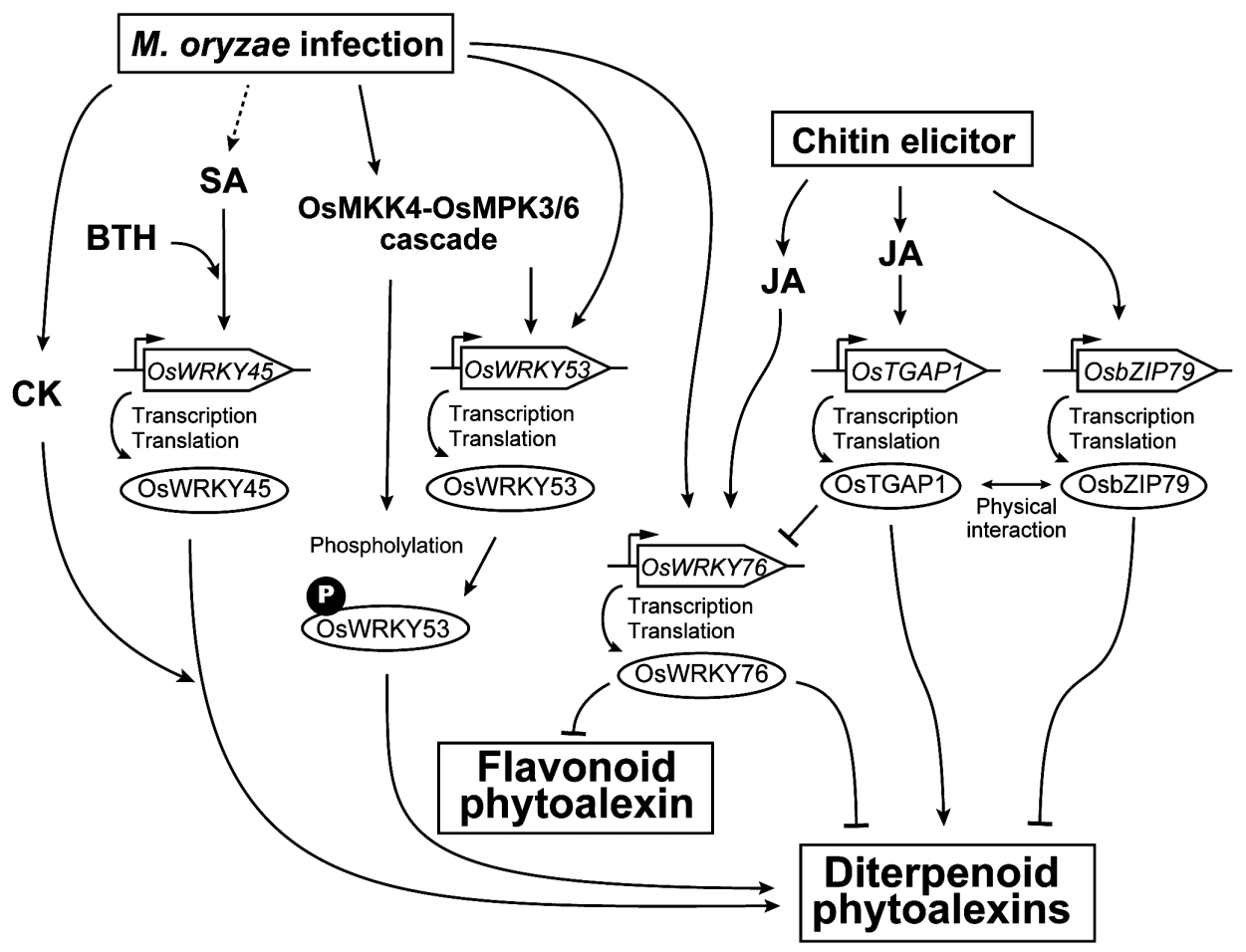

Figure 4. Transcriptional networks leading to phytoalexin production. Solid arrows represent established relationships, while dotted arrows indicate gaps in knowledge. The expression of OsTGAP1 and OsbZIP79 are induced by chitin elicitor treatment in rice cells (Okada et al. 2009). JA treatment also induces the expression of OsTGAP1. Overexpression of OsTGAP1 causes the hyperaccumulation of momilactones and phytocassanes, as well as the enhanced expression of diterpenoid phytoalexin biosynthetic genes, after treatment with an elicitor in rice cells (Miyamoto et al. 2014; Okada et al. 2009). By comparison, overexpression of OsbZIP79 suppressed the chitin elicitor-inducible expression of diterpenoid phytoalexin biosynthetic genes, causing a decrease in the accumulation of momilactones and phytocassanes in elicitor-treated rice cells (Miyamoto et al. 2015). A direct physical interaction between OsTGAP1 and OsbZIP79 has been observed (Miyamoto et al. 2015). The expression of OsWRKY76 is also induced by chitin elicitor and JA treatments in rice cells. In OsTGAP1-overexpressing rice cells, OsTGAP1 binds to the upstream region of OsWRKY76 and the chitin elicitor-induced expression of OsWRKY76 is suppressed (Miyamoto et al. 2014). The expression of OsWRKY76 is also induced by Magnaporthe oryzae infection in rice leaves. The overexpression of OsWRKY76 suppresses the accumulation of both diterpenoid phytoalexins and flavonoid phytoalexin in M. oryzae-infected rice leaves (Yokotani et al. 2013). OsWRKY53 expression is induced by M. oryzae, and OsWRKY53 protein is phosphorylated and activated by the OsMKK4-OsMPK3/OsMPK6 cascade. Overexpression of phosphomimic mutated OsWRKY53 causes the upregulation of momilactone biosynthetic genes and the slight accumulation of momilactones in the absence of pathogen attack (Chujo et al. 2014). OsWRKY45 is involved in the regulation of diterpenoid phytoalexin production in salicylic acid (SA) and cytokinin (CK) signaling pathway (Akagi et al. 2014). Benzothiadiazole (BTH) acts as a plant activator that enhances SA signaling pathway.

in OsTGAP1ox cells. We performed transcriptome analysis and chromatin immunoprecipitation with next-generation sequencing using OsTGAP1ox cells and identified OsTGAP1 target genes. None of the clustered momilactone and phytocassane biosynthetic genes was included among the OsTGAP1 target genes, suggesting that OsTGAP1 did not directly regulate the expression of these biosynthetic genes through binding to each promoter region. Interestingly, however, several OsTGAP1-binding regions were found in the intergenic regions among and near the cluster regions. Although the detailed regulatory mechanism of these clustered genes remains unknown, the binding of OsTGAP1 to the intergenic regions of the gene clusters might play a particular role in transcriptional regulation. Concerning the MEP pathway genes, only OsDXS3 possessed an OsTGAP1-binding region in its upstream region. A subsequent transactivation assay further confirmed the direct regulation of OsDXS3 expression by OsTGAP1
(Miyamoto et al. 2014).

The screening of OsTGAP1-interacting proteins using the yeast two-hybrid technique resulted in the identification of 10 candidates. Among the OsTGAP1interacting protein candidates, a TGA factor, OsbZIP79, was investigated to verify its involvement in the regulation of phytoalexin production. Intriguingly, whereas OsbZIP79 transactivation activity was observed in a transient reporter assay, the overexpression of OsbZIP79 resulted in suppression of the chitin elicitorinducible expression of diterpenoid phytoalexin biosynthetic genes, which reduced the accumulation of phytoalexin in elicitor-treated rice cells. These results suggest that OsbZIP79 functions as a negative regulator of phytoalexin production triggered by the chitin elicitor in rice cells (Miyamoto et al. 2015). The mRNA level of OsTGAP1 is induced slightly earlier than that of OsKSL4 in rice cells after chitin elicitor treatment. In contrast, the induction of the OsbZIP79 mRNA level is synchronous 
with that of the OsKSL4 mRNA level (Okada et al. 2009). Therefore, it is possible that OsbZIP79 suppresses the action of OsTGAP1 in upregulating the transcription levels of diterpenoid phytoalexin biosynthetic genes. OsTGAP1 and OsbZIP79 may act competitively to modulate the expression of diterpenoid phytoalexin biosynthetic genes, allowing fine-tuning of phytoalexin production (Miyamoto et al. 2015). Further studies should examine how the interaction of OsTGAP1 and OsbZIP79 affects elicitor-inducible phytoalexin production in rice cells.

The plant-specific zinc finger WRKY family transcription factors are also involved in phytoalexin biosynthesis. Yokotani et al. (2013) reported that in OsWRKY76-overexpressing rice plants, the inducible expression of the genes that are relevant to phytoalexin biosynthesis is suppressed, causing a decrease in the accumulation of momilactones, phytocassanes, and sakuranetin in $M$. oryzae-infected rice leaves. These results suggest that OsWRKY76 functions as a negative regulator of the production of both the diterpenoid phytoalexins and the flavonoid phytoalexin (Yokotani et al. 2013). It is also reported that OsWRKY76 and OsTGAP1 interact in the signaling pathway for diterpenoid phytoalexin production. The elicitorinduced expression of OsWRKY76 was suppressed in OsTGAP1ox cells. Taken together with the fact that OsTGAP1 was bound to the upstream region of OsWRKY76, it appears that OsTGAP1 directly downregulates the expression of OsWRKY76. The downregulation of OsWRKY76 expression is among the mechanisms explaining the enhanced expression of diterpenoid phytoalexin biosynthetic genes in OsTGAP1ox cells (Miyamoto et al. 2014). On the other hand, overexpression of OsbZIP79 did not greatly affect elicitor-induced expression of OsWRKY76, suggesting that OsbZIP79 and OsWRKY76 occupy different signaling pathways and suppress phytoalexin production in different ways (Miyamoto et al. 2015).

Another rice WRKY transcription factor, OsWRKY53, was found to be involved in the regulation of momilactone biosynthetic genes. Chujo et al. (2014) reported that OsWRKY53 was phosphorylated and activated by the OsMKK4-OsMPK3/OsMPK6 cascade (Kishi-Kaboshi et al. 2010), a fungal elicitorresponsive MAPK cascade in rice. Transgenic rice plants overexpressing a phosphomimic mutated OsWRKY53 (OsWRKY53SD) showed further enhanced disease resistance to the blast fungus, compared with native OsWRKY53-overexpressing rice plants. In addition, a substantial number of defense-related genes, including momilactone biosynthetic genes, were upregulated in the OsWRKY53SD-overexpressing plants compared with the OsWRKY53-overexpressing plants. OsWRKY53SDoverexpressing plants also exhibit a slight accumulation of momilactones even in the absence of a pathogen attack (Chujo et al. 2014). Considering that activation of the OsMKK4-OsMPK3/OsMPK6 cascade leads to the accumulation of diterpenoid phytoalexins (KishiKaboshi et al. 2010), OsWRKY53 may be one of the components of downstream signaling of this cascade.

Recently, Akagi et al. (2014) reported that the rice WRKY transcription factor, OsWRKY45, is involved in the regulation of diterpenoid phytoalexin production in SA/CK signaling pathway. OsWRKY45 plays a central role in the priming of diterpenoid phytolalexin biosynthetic genes in BTH-treated rice leaves. They also showed that the diterpenoid phytoalexin biosynthetic genes are up-regulated by the treatment of both SA and CK in a OsWRKY45-dependent manner (Akagi et al. 2014).

\section{Conclusion and perspective}

Concentrated efforts have revealed the relevant factors and signaling pathways that are involved in the regulation of phytoalexin production in rice. It is still unknown, however, how these transcription factors regulate phytoalexin biosynthetic genes in concert with one another. Further research to investigate the molecular mechanisms of transcriptional regulation of phytoalexin biosynthetic genes and to reveal how upstream signals activate each transcription factor in the signaling cascade is essential. Such work will elucidate the full picture of endogenous signaling that leads to the biosynthesis of phytoalexins.

Our current understanding, based on the evidence showing the defensive function of phytoalexins in rice, does not fully explain why rice produces this specialized metabolite. The biological roles of phytoalexins, should be investigated in more detail by characterizing mutants that are defective in the production of phytoalexin. Recently, loss-of-function mutants for OsCPS2 and OsKSL4, which are defective in momilactone, were analyzed to assess the endogenous function of momilactones (Toyomasu et al. 2014; Xu et al. 2012). In a study, the OsCPS2 mutant exhibited increased susceptibility to the blast fungus, but this effect was not seen in the case of the OsKSL4 mutant. With regard to allelopathic activity, neither of the mutants displayed an allelopathic effect on nearby weeds (Toyomasu et al. 2014; Xu et al. 2012).

So far, there has been no attempt to examine the effect of overproduction of the phytoalexins on the above biological responses in rice. The development of transgenic overexpression lines would enable the study of plants that are resistant to pathogens and suppress of the growth of weeds and would represent progress toward a chemical-free model of agriculture. We hope that the knowledge of the transcriptional regulation 
of phytoalexins synthesis will be applied to beneficial strategies in an environmentally friendly agricultural system.

\section{Acknowledgements}

We thank Dr. Hisakazu Yamane, emeritus professor of The University of Tokyo, for his support of this study. This work was supported by a JSPS Grant-in-Aid for Scientific Research (No. 22380066, 23580145), a Grant-in-Aid for JSPS Fellows (No. 09J01084), and the Program for Promotion of Basic Research Activities for Innovative Biosciences (PROBRAIN).

\section{References}

Ahuja I, Kissen R, Bones AM (2012) Phytoalexins in defense against pathogens. Trends Plant Sci 17: 73-90

Akagi A, Fukushima S, Okada K, Jiang CJ, Yoshida R, Nakayama A, Shimono M, Sugano S, Yamane H, Takatsuji H (2014) WRKY45dependent priming of diterpenoid phytoalexin biosynthesis in rice and the role of cytokinin in triggering the reaction. Plant Mol Biol 86: 171-183

Akatsuka T, Kodama O, Kato H, Sekido H, Kono Y, Takeuchi S (1983) 3-hydroxy-7-oxo-sandaracopimaradiene (oryzalexin A), a new phytoalexin isolated from rice blast leaves. Agric Biol Chem 47: 445-447

Akatsuka T, Kodama O, Sekido H, Kono Y, Takeuchi S (1985) Novel Phytoalexins (Oryzalexins A, B and C) Isolated from Rice Blast Leaves Infected with Pyricularia oryzae. Part I: Isolation, characterization and biological activities of oryzalexins. Agric Biol Chem 49: 1689-1694

Asahina Y (1908) Ueber das Sakuranin, ein neues Glykosid der Rinde von Prunus Pseudo-Cerasus Lindl. var. Sieboldi Maxim. Arch Pharm (Weinheim) 246: 259-272

Atawong A, Hasegawa M, Kodama O (2002) Biosynthesis of rice phytoalexin: Enzymatic conversion of $3 \beta$-Hydroxy- $9 \beta$-pimara7,15-dien-19,6 $\beta$-olide to momilactone A. Biosci Biotechnol Biochem 66: 566-570

Cai Q, Yuan Z, Chen M, Yin C, Luo Z, Zhao X, Liang W, Hu J, Zhang D (2014) Jasmonic acid regulates spikelet development in rice. Nat Commun 5: 3476

Cartwright D, Langcake P, Pryce R, Leworthy D, Ride J (1977) Chemical activation of host defence mechanisms as a basis for crop protection. Nature 267: 511-513

Cartwright D, Langcake P, Pryce R, Leworthy D, Ride J (1981) Isolation and characterization of two phytoalexins from rice as momilactones A and B. Phytochemistry 20: 535-537

Cho EM, Okada A, Kenmoku H, Otomo K, Toyomasu T, Mitsuhashi W, Sassa T, Yajima A, Yabuta G, Mori K, et al. (2004) Molecular cloning and characterization of a cDNA encoding entcassa-12,15-diene synthase, a putative diterpenoid phytoalexin biosynthetic enzyme, from suspension-cultured rice cells treated with a chitin elicitor. Plant J 37: 1-8

Choi J, Huh SU, Kojima M, Sakakibara H, Paek KH, Hwang I (2010) The cytokinin-activated transcription factor ARR2 promotes plant immunity via TGA3/NPR1-dependent salicylic acid signaling in Arabidopsis. Dev Cell 19: 284-295

Chu HY, Wegel E, Osbourn A (2011) From hormones to secondary metabolism: the emergence of metabolic gene clusters in plants. Plant J 66: 66-79

Chujo T, Miyamoto K, Ogawa S, Masuda Y, Shimizu T, KishiKaboshi M, Takahashi A, Nishizawa Y, Minami E, Nojiri H, et al.
(2014) Overexpression of phosphomimic mutated OsWRKY53 leads to enhanced blast resistance in rice. PLoS ONE 9: e98737

Daw BD, Zhang LH, Wang ZZ (2008) Salicylic acid enhances antifungal resistance to Magnaporthe grisea in rice plants. Aust Plant Path 37: 637-644

De Vleesschauwer D, Gheysen G, Höfte M (2013) Hormone defense networking in rice: tales from a different world. Trends Plant Sci 18: 555-565

Duan L, Liu H, Li X, Xiao J, Wang S (2014) Multiple phytohormones and phytoalexins are involved in disease resistance to Magnaporthe oryzae invaded from roots in rice. Physiol Plant 152: 486-500

Estévez JM, Cantero A, Reindl A, Reichler S, León P (2001) 1-Deoxy-D-xylulose-5-phosphate Synthase, a Limiting Enzyme for Plastidic Isoprenoid Biosynthesis in Plants. J Biol Chem 276: 22901-22909

Großkinsky DK, Naseem M, Abdelmohsen UR, Plickert N, Engelke T, Griebel T, Zeier J, Novák O, Strnad M, Pfeifhofer H, et al. (2011) Cytokinins mediate resistance against pseudomonas syringae in Tobacco through increased antimicrobial phytoalexin synthesis independent of salicylic acid signaling. Plant Physiol 157: 815-830

Hasegawa M, Mitsuhara I, Seo S, Imai T, Koga J, Okada K, Yamane $\mathrm{H}$, Ohashi Y (2010) Phytoalexin accumulation in the interaction between rice and the blast fungus. Mol Plant Microbe Interact 23: 1000-1011

Inoue Y, Sakai M, Yao Q, Tanimoto Y, Toshima H, Hasegawa M (2013) Identification of a novel casbane-type diterpene phytoalexin, ent-10-oxodepressin, from rice leaves. Biosci Biotechnol Biochem 77: 760-765

Jiang CJ, Shimono M, Sugano S, Kojima M, Liu X, Inoue H, Sakakibara H, Takatsuji H (2013) Cytokinins act synergistically with salicylic acid to activate defense gene expression in rice. $\mathrm{Mol}$ Plant Microbe Interact 26: 287-296

Kanno Y, Otomo K, Kenmoku H, Mitsuhashi W, Yamane H, Oikawa H, Toshima H, Matsuoka M, Sassa T, Toyomasu T (2006) Characterization of a rice gene family encoding type-A diterpene cyclases. Biosci Biotechnol Biochem 70: 1702-1710

Kato H, Kodama O, Akatsuka T (1993) Oryzalexin E, a diterpene phytoalexin from UV-irradiated rice leaves. Phytochemistry 33: 79-81

Kato H, Kodama O, Akatsuka T (1994) Oryzalexin F, a diterpene phytoalexin from UV-irradiated rice leaves. Phytochemistry 36: 299-301

Kato H, Kodama O, Akatsuka T (1995) Characterization of an inducible p450 hydroxylase involved in the rice diterpene phytoalexin biosynthetic pathway. Arch Biochem Biophys 316: 707-712

Kato-Noguchi H, Ino T (2003) Rice seedlings release momilactone $\mathrm{B}$ into the environment. Phytochemistry 63: 551-554

Ke Y, Liu H, Li X, Xiao J, Wang S (2014) Rice OsPAD4 functions differently from Arabidopsis AtPAD4 in host-pathogen interactions. Plant J 78: 619-631

Kishi-Kaboshi M, Okada K, Kurimoto L, Murakami S, Umezawa T, Shibuya N, Yamane H, Miyao A, Takatsuji H, Takahashi A, et al. (2010) A rice fungal MAMP-responsive MAPK cascade regulates metabolic flow to antimicrobial metabolite synthesis. Plant $J$ 63: 599-612

Ko KW, Okada K, Koga J, Jikumaru Y, Nojiri H, Yamane H (2010) Effects of cytokinin on production of diterpenoid phytoalexins in rice. J Pestic Sci 35: 412-418

Kodama O, Miyakawa J, Akatsuka T, Kiyosawa S (1992a) 
Sakuranetin, a flavanone phytoalexin from ultraviolet-irradiated rice leaves. Phytochemistry 31: 3807-3809

Kodama O, Li WX, Tamogami S, Akatsuka T (1992b) Oryzalexin $\mathrm{S}$, a novel stemarane-type diterpene rice phytoalexin. Biosci Biotechnol Biochem 56: 1002-1003

Koga J, Shimura M, Oshima K, Ogawa N, Yamauchi T, Ogasawara N (1995) Phytocassanes A, B, C and D, novel diterpene phytoalexins from rice, Oryza sativa L. Tetrahedron 51: 7907-7918

Koga J, Ogawa N, Yamauchi T, Kikuchi M, Ogasawara N, Shimura M (1997) Functional moiety for the antifungal activity of phytocassane $\mathrm{E}$, a diterpene phytoalexin from rice. Phytochemistry 44: 249-253

Koga J, Kubota H, Gomi S, Umemura K, Ohnishi M, Kono T (2006) Cholic acid, a bile acid elicitor of hypersensitive cell death, pathogenesis-related protein synthesis, and phytoalexin accumulation in rice. Plant Physiol 140: 1475-1483

Kono Y, Takeuchi S, Kodama O, Akatsuka T (1984) Absolute configuration of oryzalexin A and structures of its related phytoalexins isolated from rice blast leaves infected with Pyricularia oryzae. Agric Biol Chem 48: 253-255

Kono Y, Takeuchi S, Kodama O, Sekido H, Akatsuka T (1985) Novel phytoalexins (oryzalexins A, B and C) isolated from rice blast leaves infected with Pyricularia oryzae. Part II: Structural studies of oryzalexins. Agric Biol Chem 49: 1695-1701

Kono Y, Uzawa J, Kobayashi K, Suzuki Y, Uramoto M, Sakurai A, Watanabe M, Teraoka T, Hosokawa D, Watanabe M, et al. (1991) Structures of Oryzalides A and B, and Oryzalic Acid A, a group of novel antimicrobial diterpenes, isolated from healthy leaves of a bacterial leaf blight-resistant cultivar of rice plant. Agric Biol Chem 55: 803-811

Kono Y, Kojima A, Nagai R, Watanabe M, Kawashima T, Onizawa T, Teraoka T, Watanab M, Koshino H, Uzawa J, et al. (2004) Antibacterial diterpenes and their fatty acid conjugates from rice leaves. Phytochemistry 65: 1291-1298

Lichtenthaler HK, Schwender J, Disch A, Rohmer M (1997) Biosynthesis of isoprenoids in higher plant chloroplasts proceeds via a mevalonate-independent pathway. FEBS Lett 400: 271-274

Lin F, Yamano G, Hasegawa M, Anzai H, Kawasaki S, Kodama O (1996) Cloning and functional analysis of caffeic acid 3-Omethyltransferase from rice (Oryza sativa). J Pestic Sci 31: 47-53

Miyamoto K, Shimizu T, Lin F, Sainsbury F, Thuenemann E, Lomonossoff G, Nojiri H, Yamane H, Okada K (2012) Identification of an E-box motif responsible for the expression of jasmonic acid-induced chitinase gene OsChia4a in rice. J Plant Physiol 169: 621-627

Miyamoto K, Matsumoto T, Okada A, Komiyama K, Chujo T, Yoshikawa H, Nojiri H, Yamane H, Okada K (2014) Identification of target genes of the bZIP transcription factor OsTGAP1, whose overexpression causes elicitor-induced hyperaccumulation of diterpenoid phytoalexins in rice cells. PLoS ONE 9: e105823

Miyamoto K, Nishizawa Y, Minami E, Nojiri H, Yamane H, Okada K (2015) Overexpression of the bZIP transcription factor OsbZIP79 suppresses the production of diterpenoid phytoalexin in rice cells. J Plant Physiol 173: 19-27

Nakazato Y, Tamogami S, Kawai H, Hasegawa M, Kodama O (2000) Methionine-induced phytoalexin production in rice leaves. Biosci Biotechnol Biochem 64: 577-583

Nemoto T, Cho EM, Okada A, Okada K, Otomo K, Kanno Y, Toyomasu T, Mitsuhashi W, Sassa T, Minami E, et al. (2004) Stemar-13-ene synthase, a diterpene cyclase involved in the biosynthesis of the phytoalexin oryzalexin S in rice. FEBS Lett 571: 182-186

Nojiri H, Sugimori M, Yamane H, Nishimura Y, Yamada A, Shibuya N, Kodama O, Murofushi N, Omori T (1996) Involvement of jasmonic acid in elicitor-induced phytoalexin production in suspension-cultured rice cells. Plant Physiol 110: 387-392

Nützmann HW, Osbourn A (2014) Gene clustering in plant specialized metabolism. Curr Opin Biotechnol 26: 91-99

Okada A, Shimizu T, Okada K, Kuzuyama T, Koga J, Shibuya N, Nojiri H, Yamane H (2007) Elicitor induced activation of the methylerythritol phosphate pathway toward phytoalexins biosynthesis in rice. Plant Mol Biol 65: 177-187

Okada A, Okada K, Miyamoto K, Koga J, Shibuya N, Nojiri H, Yamane H (2009) OsTGAP1, a bZIP transcription factor, coordinately regulates the inductive production of diterpenoid phytoalexins in rice. J Biol Chem 284: 26510-26518

Okada K (2011) The biosynthesis of isoprenoids and the mechanisms regulating it in plants. Biosci Biotechnol Biochem 75: $1219-1225$

Otomo K, Kanno Y, Motegi A, Kenmoku H, Yamane H, Mitsuhashi W, Oikawa H, Toshima H, Itoh H, Matsuoka M, et al. (2004a) Diterpene cyclases responsible for the biosynthesis of phytoalexins, momilactones $\mathrm{A}, \mathrm{B}$, and oryzalexins $\mathrm{A}-\mathrm{F}$ in rice. Biosci Biotechnol Biochem 68: 2001-2006

Otomo K, Kenmoku H, Oikawa H, Konig WA, Toshima H, Mitsuhashi W, Yamane H, Sassa T, Toyomasu T (2004b) Biological functions of ent- and syn-copalyl diphosphate synthases in rice: Key enzymes for the branch point of gibberellin and phytoalexin biosynthesis. Plant J 39: 886-893

Park HL, Lee SW, Jung KH, Hahn TR, Cho MH (2013) Transcriptomic analysis of UV-treated rice leaves reveals UVinduced phytoalexin biosynthetic pathways and their regulatory networks in rice. Phytochemistry 96: 57-71

Peters RJ (2006) Uncovering the complex metabolic network underlying diterpenoid phytoalexin biosynthesis in rice and other cereal crop plants. Phytochemistry 67: 2307-2317

Prisic S, Xu M, Wilderman PR, Peters RJ (2004) Rice contains two disparate ent-copalyl diphosphate synthases with distinct metabolic functions. Plant Physiol 136: 4228-4236

Rakwal R, Hasegawa M, Kodama O (1996a) A methyltransferase for synthesis of the flavanone phytoalexin sakuranetin in rice leaves. Biochem Biophys Res Commun 222: 732-735

Rakwal R, Tamogami S, Kodama O (1996b) Role of jasmonic acid as a signaling molecule in copper chloride-elicited rice phytoalexin production. Biosci Biotechnol Biochem 60: 1046-1048

Rakwal R, Agrawal GK, Yonekura M, Kodama O (2000) Naringenin 7-O-methyltransferase involved in the biosynthesis of the flavanone phytoalexin sakuranetin from rice (Oryza sativa L.). Plant Sci 155: 213-221

Riemann M, Haga K, Shimizu T, Okada K, Ando S, Mochizuki S, Nishizawa Y, Yamanouchi U, Nick P, Yano M, et al. (2013) Identification of rice ALLENE OXIDE CYCLASE mutants and the function of jasmonate for defence against Magnaporthe oryzae. Plant J 74: 226-238

Sakamoto T, Miura K, Itoh H, Tatsumi T, Ueguchi-Tanaka M, Ishiyama K, Kobayashi M, Agrawal GK, Takeda S, Abe K, et al. (2004) An overview of gibberellin metabolism enzyme genes and their related mutants in rice. Plant Physiol 134: 1642-1653

Schmelz EA, Huffaker A, Sims JW, Christensen SA, Lu X, Okada K, Peters RJ (2014) Biosynthesis, elicitation and roles of monocot terpenoid phytoalexins. Plant J 79: 659-678

Sekido H, Endo T, Suga R, Kodama O, Akatsuka T, Kono 
Y, Takeuchi S (1986) Oryzalexin D (3,7-dihydroxy-(+)sandaracopimaradiene), a new phytoalexin isolated from blastinfected rice leaves. J Pestic Sci 11: 369-372

Shimizu T, Jikumaru Y, Okada A, Okada K, Koga J, Umemura K, Minami E, Shibuya N, Hasegawa M, Kodama O, et al. (2008) Effects of a bile acid elicitor, cholic acid, on the biosynthesis of diterpenoid phytoalexins in suspension-cultured rice cells. Phytochemistry 69: 973-981

Shimizu T, Nakano T, Takamizawa D, Desaki Y, Ishii-Minami N, Nishizawa Y, Minami E, Okada K, Yamane H, Kaku H, et al. (2010) Two LysM receptor molecules, CEBiP and OsCERK1, cooperatively regulate chitin elicitor signaling in rice. Plant $J$ 64: 204-214

Shimizu T, Lin F, Hasegawa M, Okada K, Nojiri H, Yamane H (2012) Purification and identification of naringenin 7-Omethyltransferase, a key enzyme in biosynthesis of flavonoid phytoalexin sakuranetin in rice. J Biol Chem 287: 19315-19325

Shimizu T, Miyamoto K, Miyamoto K, Minami E, Nishizawa Y, Iino M, Nojiri H, Yamane H, Okada K (2013) OsJAR1 contributes mainly to biosynthesis of the stress-induced jasmonoylisoleucine involved in defense responses in rice. Biosci Biotechnol Biochem 77: 1556-1564

Shimura K, Okada A, Okada K, Jikumaru Y, Ko KW, Toyomasu T, Sassa T, Hasegawa M, Kodama O, Shibuya N, et al. (2007) Identification of a biosynthetic gene cluster in rice for momilactones. J Biol Chem 282: 34013-34018

Swaminathan S, Morrone D, Wang Q, Fulton DB, Peters RJ (2009) CYP76M7 is an ent-cassadiene C11alpha-hydroxylase defining a second multifunctional diterpenoid biosynthetic gene cluster in rice. Plant Cell 21: 3315-3325

Tamogami S, Rakwal R, Kodama O (1997) Phytoalexin production elicited by exogenously applied jasmonic acid in rice leaves (Oryza sativa L.) is under the control of cytokinins and ascorbic acid. FEBS Lett 412: 61-64

Taniguchi S, Hosokawa-Shinonaga Y, Tamaoki D, Yamada S, Akimitsu K, Gomi K (2014a) Jasmonate induction of the monoterpene linalool confers resistance to rice bacterial blight and its biosynthesis is regulated by JAZ protein in rice. Plant Cell Environ 37: 451-461

Taniguchi S, Miyoshi S, Tamaoki D, Yamada S, Tanaka K, Uji Y, Tanaka S, Akimitsu K, Gomi K (2014b) Isolation of jasmonateinduced sesquiterpene synthase of rice: Product of which has an antifungal activity against Magnaporthe oryzae. J Plant Physiol 171: 625-632

Toyomasu T (2008) Recent advances regarding diterpene cyclase genes in higher plants and fungi. Biosci Biotechnol Biochem 72: 1168-1175

Toyomasu T, Kagahara T, Okada K, Koga J, Hasegawa M, Mitsuhashi W, Sassa T, Yamane H (2008) Diterpene phytoalexins are biosynthesized in and exuded from the roots of rice seedlings. Biosci Biotechnol Biochem 72: 562-567

Toyomasu T, Usui M, Sugawara C, Otomo K, Hirose Y, Miyao A, Hirochika H, Okada K, Shimizu T, Koga J, et al. (2014) Reverse-genetic approach to verify physiological roles of rice phytoalexins: characterization of a knockdown mutant of OsCPS4 phytoalexin biosynthetic gene in rice. Physiol Plant 150: 55-62
Vranová E, Coman D, Gruissem W (2013) Network analysis of the MVA and MEP pathways for isoprenoid synthesis. Annu Rev Plant Biol 64: 665-700

Wang Q, Hillwig ML, Peters RJ (2011) CYP99A3: functional identification of a diterpene oxidase from the momilactone biosynthetic gene cluster in rice. Plant J 65: 87-95

Wang Q, Hillwig ML, Okada K, Yamazaki K, Wu Y, Swaminathan S, Yamane H, Peters RJ (2012a) Characterization of CYP76M5-8 indicates metabolic plasticity within A plant biosynthetic gene cluster. J Biol Chem 287: 6159-6168

Wang Q, Hillwig ML, Wu Y, Peters RJ (2012b) CYP701A8: a rice ent-kaurene oxidase paralog diverted to more specialized diterpenoid metabolism. Plant Physiol 158: 1418-1425

Wasternack C, Hause B (2013) Jasmonates: biosynthesis, perception, signal transduction and action in plant stress response, growth and development. An update to the 2007 review in Annals of Botany. Ann Bot (Lond) 111: 1021-1058

Watanabe M, Sakai Y, Teraoka T, Abe H, Kono Y, Uzawa J, Kobayashi K, Suzuki Y, Sakurai A (1990) Novel C19-kaurane type of diterpene (Oryzalide A), a new antimicrobial compound isolated from healthy leaves of a bacterial leaf blight-resistant cultivar of rice plant. Agric Biol Chem 54: 1103-1105

Watanabe M, Kono Y, Watanabe M, Uzawa J, Teraoka T, Hosokawa D, Suzuki Y, Sakurai A, Teraguchi M (1992) Structures of Oryzalic acid B and three related compounds, a group of novel antibacterial diterpenes, isolated from leaves of a bacterial leaf blight-resistent cultivar of rice. Biosci Biotechnol Biochem 56: 113-117

Wilderman PR, Xu M, Jin Y, Coates RM, Peters RJ (2004) Identification of syn-pimara-7,15-diene synthase reveals functional clustering of terpene synthases involved in rice phytoalexin/allelochemical biosynthesis. Plant Physiol 135: 2098-2105

Wu Y, Hillwig ML, Wang Q, Peters RJ (2011) Parsing a multifunctional biosynthetic gene cluster from rice: biochemical characterization of CYP71Z6 \& 7. FEBS Lett 585: 3446-3451

Xu M, Galhano R, Wiemann P, Bueno E, Tiernan M, Wu W, Chung IM, Gershenzon J, Tudzynski B, Sesma A, et al. (2012) Genetic evidence for natural product-mediated plant-plant allelopathy in rice (Oryza sativa). New Phytol 193: 570-575

Xu M, Hillwig ML, Prisic S, Coates RM, Peters RJ (2004) Functional identification of rice syn-copalyl diphosphate synthase and its role in initiating biosynthesis of diterpenoid phytoalexin/allelopathic natural products. Plant J 39: 309-318

Yajima A, Mori K (2000) Diterpenoid total synthesis, XXXII Synthesis and absolute configuration of (-)-phytocassane D, a diterpene phytoalexin isolated from the rice plant, Oryza sativa. Eur J Org Chem 2000: 4079-4091

Ye M, Luo S, Xie J, Li Y, Xu T, Liu Y, Song Y, Zhu-Salzman K, Zeng R (2012) Silencing COI1 in rice increases susceptibility to chewing insects and impairs inducible defense. PLoS ONE 4: e36214

Yokotani N, Sato Y, Tanabe S, Chujo T, Shimizu T, Okada K, Yamane H, Shimono M, Sugano S, Takatsuji H, et al. (2013) WRKY76 is a rice transcriptional repressor playing opposite roles in blast disease resistance and cold stress tolerance. J Exp Bot 64: 5085-5097 